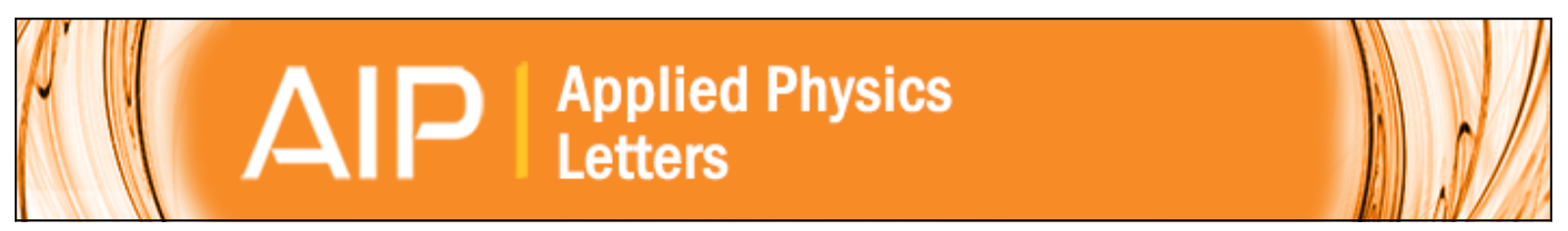

\title{
Passive optical enhancement of laser-microwave synchronization
}

A. Gliserin, M. Walbran, and P. Baum

Citation: Applied Physics Letters 103, 031113 (2013); doi: 10.1063/1.4815929

View online: http://dx.doi.org/10.1063/1.4815929

View Table of Contents: http://scitation.aip.org/content/aip/journal/apl/103/3?ver=pdfcov

Published by the AIP Publishing

\section{Advertisement:}
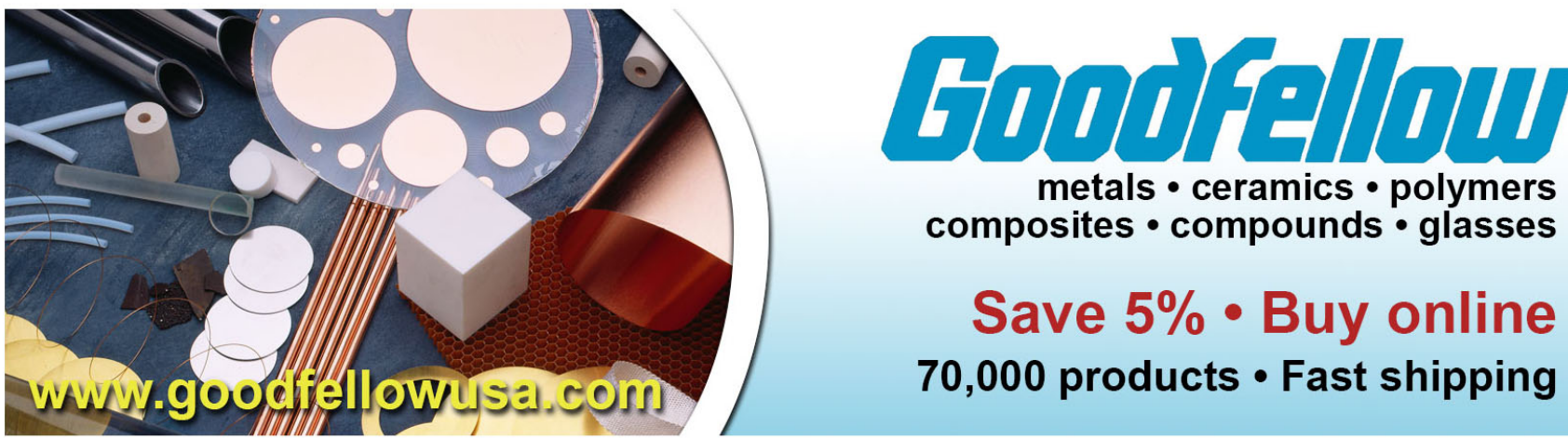

metals • ceramics • polymers composites • compounds • glasses

Save $5 \% \cdot$ Buy online 70,000 products $\cdot$ Fast shipping 


\title{
Passive optical enhancement of laser-microwave synchronization
}

\author{
A. Gliserin, M. Walbran, and P. Baum ${ }^{\text {a) }}$ \\ Max-Planck-Institute of Quantum Optics, and Ludwig-Maximilians-Universität München, Am Coulombwall 1, \\ 85748 Garching, Germany
}

(Received 13 May 2013; accepted 30 June 2013; published online 17 July 2013)

\begin{abstract}
Thermal noise is a fundamental limitation for synchronizing microwaves to high-power lasers of low repetition rate. Here, we describe an optical enhancement scheme that concentrates the output power of a fast photodiode into a narrow range of harmonics around a microwave frequency. The scheme is entirely passive and requires no feedback or lock. Using a 5-MHz laser and a microwave at $6.2 \mathrm{GHz}$, we demonstrate an enhancement of optical-to-microwave conversion by a factor of 4000. The uncorrelated noise on time scales up to 8 min amounts to less than $4 \mathrm{fs}$, with laser pulses intense enough for pump-probe experiments of structural dynamics. (C) 2013 AIP Publishing LLC. [http://dx.doi.org/10.1063/1.4815929]
\end{abstract}

Time-resolved experiments with ultrashort electron pulses, synchrotrons, or free-electron lasers require a precise synchronization of the microwaves involved in the particle or X-ray bunching with the timing of a femtosecond laser, ${ }^{1}$ which is intense enough to excite the investigated dynamics in the sample. Laser-microwave synchronization with subfemtosecond jitter was demonstrated with mode-locked oscillators at repetition rates of $75 \mathrm{MHz}$ and above, ${ }^{1-4}$ but the pulse energy of these laser systems is insufficient for initiating structural dynamics for an electron or X-ray diffraction experiment. The required pulse energies on the $\mu \mathrm{J}$ level can only be generated at lower repetition rates in the $\mathrm{kHz}$ to few-MHz regime. Synchronization of microwaves to such lasers suffers from the huge difference of time scales: The period of a microwave is tens to hundreds of picoseconds, while the delay between intense laser pulses is hundreds of nanoseconds or microseconds. For a lock with femtosecond or attosecond accuracy, a relative precision of $10^{-5}$ is hence inevitable.

In most synchronization approaches, ${ }^{3-6}$ with the notable exception of the optical mixer, ${ }^{2}$ a fast photodiode is used as a primary measure of the laser's timing. A photodiode illuminated by femtosecond laser pulses generates photocurrent pulses containing modes at harmonic orders of the laser's repetition rate up to the photodiode's cutoff frequency (in the $\mathrm{GHz}$ range for fast photodiodes). ${ }^{7,8}$ Typically, one mode in the microwave range is filtered from the photodiode's output signal to be used for synchronization applications.

Thermal noise is a fundamental limitation to the quality of synchronization that can be achieved. ${ }^{6,9}$ With a given power $P_{\text {microwave }}$ of the electrical signal at a microwave frequency $f_{\text {microwave }}$, the best possible root-mean-square timing jitter $J$ of a system within a bandwidth $\Delta f$ is limited by ${ }^{10}$

$$
J \approx \frac{1}{2 \pi f_{\text {microwave }}} \sqrt{\frac{-174 \mathrm{dBm} / \mathrm{Hz}}{P_{\text {microwave }}} \Delta f},
$$

where the $-174 \mathrm{dBm} / \mathrm{Hz}\left(4 \times 10^{-21} \mathrm{~W} / \mathrm{Hz}\right)$ describes the thermal white noise at room temperature, caused by random

\footnotetext{
${ }^{\text {a) }}$ Author to whom correspondence should be addressed. Electronic mail: peter.baum@lmu.de
}

agitation of charge carriers. For example, at $6.2 \mathrm{GHz}$ and for a bandwidth of $1 \mathrm{MHz}, P_{\text {microwave }}$ must be larger than $-26 \mathrm{dBm}(2.5 \mu \mathrm{W})$ for possibly achieving sub-fs jitter. For lasers of low repetition rate, such high average power levels at $\mathrm{GHz}$ frequencies cannot be obtained, as fast photodiodes are limited by saturation effects when illuminated with intense laser pulses. ${ }^{5,11}$ In addition, the power is usually nearly equally distributed over a very large number of narrow harmonics. ${ }^{7,8}$ Therefore, only a small fraction of the total output power is available at the desired microwave mode. These two effects make it difficult to achieve a sufficiently high power level above thermal noise required for sub-fs jitter.

Here we demonstrate a passive optical element that redistributes the photodiode's output power into those harmonics desired for microwave synchronization. Figure 1 depicts our approach. An optical cavity of two curved mirrors with a distance $\mathrm{L}$ converts each incoming laser pulse into a decaying series of pulses with a temporal spacing corresponding to the desired microwave frequency. In the frequency domain, this constitutes a band-pass filter with a central passband frequency $f_{\text {microwave }}=c /(2 L)$, so that those modes of the incoming laser's frequency comb located around multiples of $f_{\text {microwave }}$ are transmitted, while others are suppressed. This effectively enhances the photodiode's output power at $f_{\text {microwave }}$ as compared to unfiltered illumination with the same optical power.

Our optical cavity resembles a passive version of some repetition-rate multipliers reported before. ${ }^{5,6,12-14}$ The FabryPérot cavities there are operated at very high laser repetition rates (hundreds of $\mathrm{MHz}$ to $1 \mathrm{GHz}$ ), so that the incoming laser pulses interfere constructively with the pulse oscillating inside the cavity, thus requiring an interferometric stabilization by means of an active feedback loop. In contrast, the pulse oscillating inside our passive filter is optically depleted before the next laser pulse arrives; hence the scheme is passive and no feedback or lock is needed. Still, the enhancement of microwave power is more than three orders of magnitude and lifts the microwave signal sufficiently high above thermal noise for achieving sub-femtosecond synchronization with lasers of rather low repetition rate. Our scheme differs from cascaded Mach-Zehnder interferometers ${ }^{6,15}$ by 


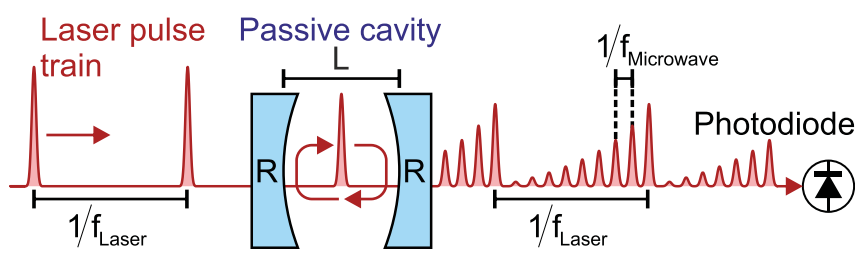

FIG. 1. Optical enhancement of microwave generation from a laser with low repetition rate. A passive optical cavity (blue) produces a decaying pulse train (red) out of each incoming laser pulse. The separation $\mathrm{L}$ of the mirrors is inversely proportional to the center frequency of the enhancement of the photodiode output at a microwave frequency.

producing a significantly higher multiplication factor, here about 1200 as compared to 8-20 there. ${ }^{6,15}$

The enhancement of the microwave signal by our passive optical mode filter depends on the reflectivity $R$ of the two mirrors; there is an optimum choice. If $R$ is too large, the back reflection from the first mirror makes it difficult to couple in enough laser power. On the other hand, if $R$ is too low, the pulse trains decay too fast, reducing the enhancement effect. The optimum reflectivity is found by considering the Fourier transform of the pulse train, taking into account the photodiode's finite response time. The intensity $I_{N}$ of the $N$-th pulse is given by $I_{N} \propto(1-R)^{2} R^{2(N-1)}$. We numerically simulated such a decaying pulse train in the time domain for a laser repetition rate of $5.1 \mathrm{MHz}$, a photodiode response time of $60 \mathrm{ps}$ (full width at half maximum), and a mirror separation of $24 \mathrm{~mm}$, corresponding to a microwave frequency of $6.237 \mathrm{GHz}$. Figure 2 shows the power spectral density at this frequency as a function of the reflectivity of the mirrors for a constant laser power before the first mirror. An optimum is found at a reflectivity of $99.75 \%$, corresponding to a finesse of 1257 . This is the best trade-off between throughput (low reflectivity) and low decay rate (high reflectivity) of the pulse train.

Next we describe the experiment. For our studies of structural dynamics with ultrafast electron diffraction in the single-electron regime, ${ }^{16}$ we aim for generating a microwave at $6.237 \mathrm{GHz}$, used to compress single-electron pulses to few-femtosecond duration by using time-dependent acceleration fields. ${ }^{17}$ The laser system is a mode-locked long-cavity Ti:Sapphire oscillator, ${ }^{18}$ providing $0.5 \mu \mathrm{J}$ of pulse energy at $800 \mathrm{~nm}$ central wavelength at a repetition rate of $5.129 \mathrm{MHz}$ (Femtosource XL, Femtolasers Produktions GmbH). The microwave frequency is the 1216th harmonic of the laser's repetition rate.

In a first experiment, the laser pulse train is focused directly on a fast photodiode (ET4000, EOT Inc.) with an

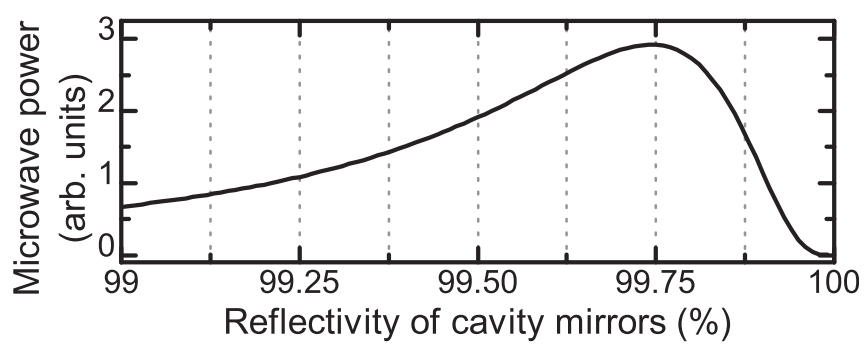

FIG. 2. Calculation of the microwave enhancement in dependence of the mirror reflectivity. incident power of $2.5 \mathrm{~mW}$. This is in the saturation regime and more input would generate less microwave power. Figure 3(a) shows the resulting spectrum, a frequency comb with a spacing of $5.1 \mathrm{MHz}$ and a power of $-51 \mathrm{dBm}$ per mode. This is insufficient to compete with thermal noise, because it would only permit a timing accuracy of 18 fs per $1 \mathrm{MHz}$ of bandwidth according to Eq. (1).

In a second experiment, we applied the optical mode filter. The reflectivity is $R=(99.75 \pm 0.05) \%$, the mirror separation is $L=24 \mathrm{~mm}$, and the mirrors have a radius of curvature of $200 \mathrm{~mm}$. Transversal mode matching is achieved with an $f=200 \mathrm{~mm}$ lens. The power reflected back from the first mirror is separated from the incoming beam by a Faraday rotator and is available for the pump-probe experiment. As before, $2.5 \mathrm{~mW}$ are incident on the photodiode; this is the maximum available laser power after the mode filter and slightly below the saturation threshold of the photodiode. Figure 3(b) shows the extracted microwave spectrum after the mode filter. The power of the 1216th harmonic $(6.237 \mathrm{GHz})$ is $-15 \mathrm{dBm}$. This is an enhancement of $\sim 36 \mathrm{~dB}$ (factor of $\sim 4000$ ) as compared to the direct detection without optical mode filtering (Fig. 3(a)). According to Eq. (1), this power is sufficiently high above thermal noise and allows to achieve a jitter of less than 0.3 fs per $1 \mathrm{MHz}$ of bandwidth.

Since the optical cavity is a passive band-pass filter, we expect a $180^{\circ}$ phase flip over the bandwidth. The suppression of the sidebands at $\pm 5.1 \mathrm{MHz}$ around the central enhanced microwave frequency at $6.237 \mathrm{GHz}$ is $\sim 4 \mathrm{~dB}$, indicating a finesse of $\sim 665$. We attribute the deviation from the expected finesse (1257 for $R=99.75 \%$ ) to an imperfect modematching between the incoming beam and the filter element. The phase dependence of our optical filter was measured by mixing the $6.237 \mathrm{GHz}$ mode (1216th harmonic of the laser's repetition rate) detected by the photodiode after the cavity

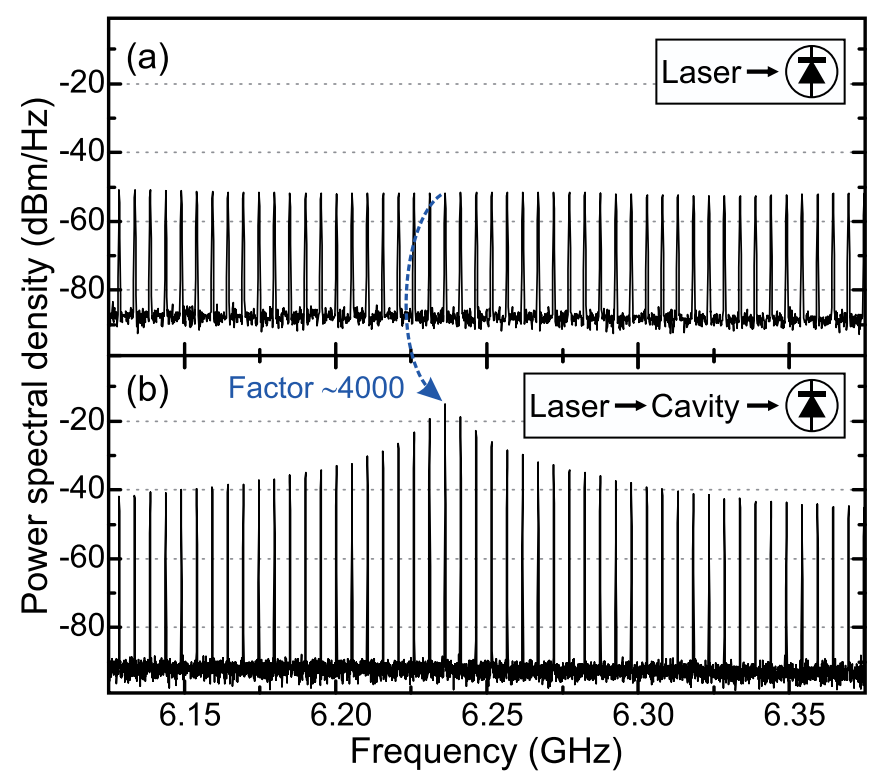

FIG. 3. Results of the microwave enhancement. (a) The photodiode's microwave spectrum at direct illumination with $2.5 \mathrm{~mW}$ of a $5 \mathrm{MHz}$ laser. (b) The photodiode's microwave spectrum using the optical enhancement system. The power of the desired mode (blue arrow) is increased by a factor of 4000 (36 dB). 
with the output of a signal generator that was phase-locked to the same harmonic without the cavity. The cavity's passband frequency was varied over a short range by changing the mirror separation. Figure 4 shows the measured phase shift (blue), together with the intensity of the comb modes around the passband frequency. The phase shows the expected change of $\pm 90^{\circ}$ over the bandwidth of about $9.5 \mathrm{MHz}$. At the central passband frequency, the slope is $16.7^{\circ} / \mathrm{MHz}$. This corresponds to a timing deviation of $7.45 \mathrm{fs} / \mathrm{kHz}$, or $1.93 \mathrm{fs} / \mathrm{nm}$ in terms of change of the cavity length.

Next we consider the performance of our scheme in the experiment. It is difficult to measure a sub-femtosecond jitter directly. What we can show here is an upper limit for the contributions to timing jitter by statistical noise, here governed by thermal noise. To this end, we measured the phase noise between two identical photodiodes illuminated by the same mode-filtered laser source via a beam splitter. Technical and systematical noise sources, for example from laser drifts, amplitude noise, or cavity vibrations are correlated and cancel out, leaving only the effects of thermal noise at the two photodiodes. The 1216th harmonic signals $(6.237 \mathrm{GHz})$ from both photodiodes are filtered, amplified and fed into a calibrated mixer. The mixer's output is lowpass filtered, amplified, and sampled by an analog-to-digital converter as well as by a spectrum analyzer. Figure 5 shows the uncorrelated single-sideband phase noise between both photodiodes at the $6.237 \mathrm{GHz}$ carrier from $2 \mathrm{mHz}(8 \mathrm{~min})$ to $1 \mathrm{MHz}$, thus covering slow drifts as well as high-frequency components. The black curve (low frequencies) is obtained from the analog-to-digital converter, while the green curve (high frequencies) is measured with the spectrum analyzer. The calculated noise floor that could at best result from direct detection is shown as the dotted line at $-126 \mathrm{dBc} / \mathrm{Hz}$. Thermal noise limits the possibly achievable jitter to $18 \mathrm{fs}$ per $1 \mathrm{MHz}$ of bandwidth in that case. The integrated uncorrelated timing jitter obtained from the experiment with mode filter is shown as the dotted blue curve. It yields $\sim 3.5$ fs over the measured frequency range, which is an upper limit given by the noise floor of the measurement system. This result demonstrates a reduction of uncorrelated thermal jitter to levels significantly below what is possible with direct detection.

The above measurement is about uncorrelated noise only, in order to study the function and benefits of our

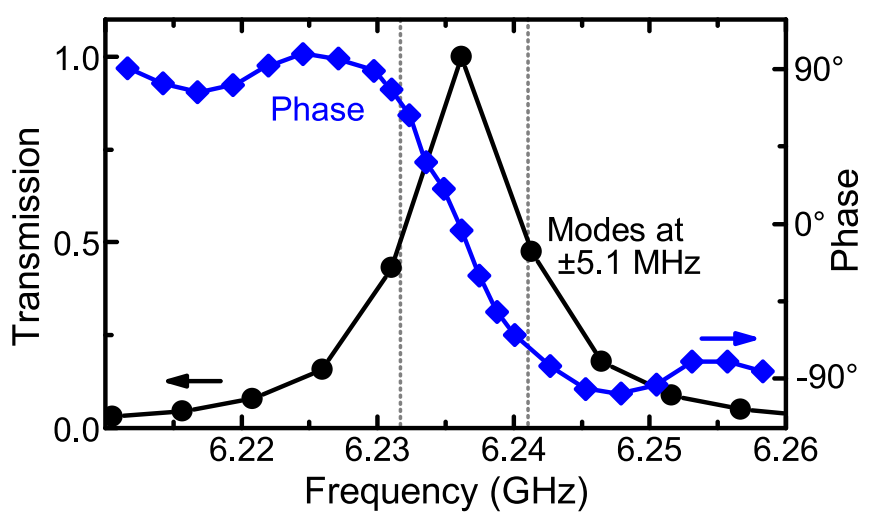

FIG. 4. Measurement of phase (blue diamonds) and bandwidth (dots) of the optical mode filter.

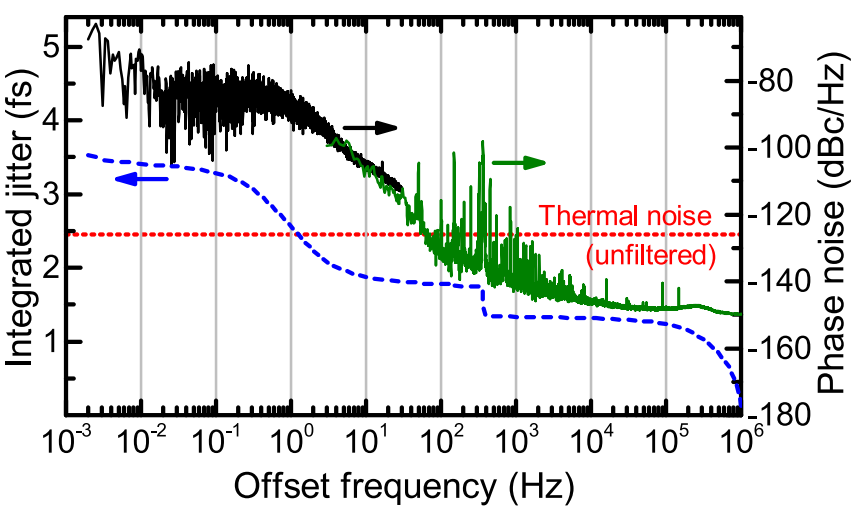

FIG. 5. Measurement of the uncorrelated single-sideband phase noise at a $6.237 \mathrm{GHz}$ carrier between two identical photodiodes after microwave enhancement with the same mode filter. The phase noise was measured with an analog-to-digital converter (black) and a spectrum analyzer (green). Above Fourier frequencies of $70 \mathrm{~Hz}$, this is significantly below the thermal noise floor without the filter (dotted red line). The integrated jitter (blue) amounts to $3.5 \mathrm{fs}$ within a frequency range of $2 \mathrm{mHz}$ to $1 \mathrm{MHz}(8 \mathrm{~min}$ to $1 \mathrm{~ns})$. There are additional contributions from correlated or systematic noise.

approach. Next, we discuss what we may expect in practice. The passive enhancement system suppresses the uncorrelated jitter due to thermal noise significantly, but systematic contributions can also degrade the quality of synchronization. First, repetition rate drifts of the laser translate into timing drifts for a passive optical filter because of its phase slope $(7.45 \mathrm{fs} / \mathrm{kHz}$ ). Our free-running laser drifts by less than $1 \mathrm{~Hz}$ over $1 \mathrm{~h},{ }^{17}$ which corresponds to about $1.2 \mathrm{kHz}$ at the 1216 th harmonic or about $9 \mathrm{fs}$ of timing drift. Likewise, the distance between the cavity mirrors must be quite stable, since a mechanical displacement of $10 \mathrm{~nm}$ already introduces about $20 \mathrm{fs}$ of timing drift. Currently, thermal drifts limit the long-term stability of our cavity's length to $20-30 \mathrm{~nm}$, corresponding to 40-60 fs. Nanometer-scaled stability is achievable with a monolithic design; appropriate materials and isolation from thermal drifts and mechanical vibrations can provide stability on picometer scales. ${ }^{19}$ With the measured $1.93 \mathrm{fs} / \mathrm{nm}$, we hence conceive that drifts of less than one femtosecond can become achievable. Second, the laser's amplitude noise also contributes to jitter. Power-to-phase couplings in photodiodes translate the fluctuations of the laser pulse energy into phase noise. ${ }^{20}$ The amplitude noise of our laser, $\Delta \mathrm{P} / \mathrm{P}_{0}$, is about $0.5 \%$ root-mean-square at good conditions. The amplitude-to-phase coefficient measured in our setup was $0.07 \mathrm{rad} /\left(\Delta \mathrm{P} / \mathrm{P}_{0}\right)$ with $2 \mathrm{~mW}$ incident on the photodiode. This yields $\sim 10$ fs of timing jitter, but this contribution can be minimized by choosing an appropriately selected photodiode and incident power. ${ }^{20,21}$ Third, fluctuations of the timing in a photocurrent peak (shot noise) can also limit the timing accuracy of photodetection. ${ }^{9}$ In our experiments, the shot noise power is orders of magnitude below the thermal noise floor and is hence insignificant. Additional technical noise is introduced whenever high microwave power and hence amplification of the photodiode's signal is needed. ${ }^{17,22}$ Here the mode filter reduces the required amplifier gain by providing a higher microwave power level directly from the photodiode.

With or without the optical enhancement, the achievable synchronization is related to the optical power incident on the 
photodiode. Devices capable of handling high photocurrent ${ }^{8,23}$ hence promise a reduced jitter. This advantage, however, diminishes with lasers of lower repetition rate (unless modefiltered), because not the average power but rather the peak power leads to saturation of the photodiode in that regime..$^{5,11}$

Let us have an outlook. Laser spectroscopy has now entered the attosecond domain, ${ }^{24-26}$ but spatial information on the atomic scale is still hidden from such experiments. Our goal with the reported laser-microwave synchronization is to advance ultrafast structural imaging by pump-probe electron diffraction into the few-femtosecond or eventually attosecond regime. ${ }^{27}$ Single-electron pulses have no space charge and can in principle be compressed to attosecond duration using the time-dependent longitudinal fields of a microwave. ${ }^{28}$ Alternatively, dense electron packets can be compressed to femtosecond duration..$^{21,29,30}$ The singleelectron source, ${ }^{16}$ the microwave compressor, ${ }^{17}$ an isochronic magnetic lens system, ${ }^{31}$ tilted excitation pulses ${ }^{32}$ and a coherent diffraction methodology ${ }^{33}$ are mostly ready; just the quality of synchronization is not at the desired fewfemtosecond level yet. With the here described approach, probably in combination with an optical mixer ${ }^{2}$ for compensating long-term drifts, we may reach into the fewfemtosecond, ultimately attosecond regime of structural dynamics.

Our scheme may also have applications in other fields. Whenever the timing of a laser needs to be detected precisely with a photodiode, an optical conversion of the laser power into convenient frequencies should be helpful for overcoming the effects of thermal and shot noise. Our passive mode filter might hence be useful as a general type of first element in any laser-microwave synchronization scheme, even if extensive feedback electronics is applied later. In the end, synchronization can only be as good as the timing of laser pulses is measurable, a feat that our passive mode filter can help with.

${ }^{1}$ J. Kim and F. X. Kärtner, Laser Photonics Rev. 4, 432-456 (2010), and references therein.

${ }^{2}$ K. Jung and J. Kim, Opt. Lett. 37, 2958 (2012).

${ }^{3}$ T. M. Fortier, M. S. Kirchner, F. Quinlan, J. Taylor, J. C. Bergquist, T. Rosenband, N. Lemke, A. Ludlow, Y. Jiang, C. W. Oates, and S. A. Diddams, Nat. Photonics 5, 425-429 (2011).

${ }^{4}$ W. Zhang, Z. Xu, M. Lours, R. Boudot, Y. Kersalé, G. Santarelli, and Y. Le Coq, Appl. Phys. Lett. 96, 211105 (2010).

${ }^{5}$ S. A. Diddams, M. Kirchner, T. Fortier, D. Braje, A. M. Weiner, and L. Hollberg, Opt. Express 17, 3331-3340 (2009).
${ }^{6}$ H. Jiang, J. Taylor, F. Quinlan, T. Fortier, and S. A. Diddams, IEEE Photon. J. 3, 1004 (2011).

${ }^{7}$ R. Sabella and S. Merli, IEEE J. Quantum Electron. 29, 906-916 (1993).

${ }^{8}$ Z. Li, H. Pan, H. Chen, A. Beling, and J. C. Campbell, IEEE J. Quantum Electron. 46, 626-632 (2010).

${ }^{9}$ F. Quinlan, T. M. Fortier, H. Jiang, A. Hati, C. Nelson, Y. Fu, J. C. Campbell, and S. A. Diddams, Nat. Photonics 7, 290-293 (2013).

${ }^{10} \mathrm{I}$. Zamek and S. Zamek, in Proceedings of IEEE International Test Conference (ITC 2005), IEEE, (2005), pp. 25-34.

${ }^{11}$ K. J. Williams, R. D. Esman, and M. Dagenais, IEEE Photon. Technol. Lett. 6, 639 (1994).

${ }^{12}$ M. S. Kirchner, D. A. Braje, T. M. Fortier, A. M. Weiner, L. Hollberg, and S. A. Diddams, Opt. Lett. 34, 872 (2009).

${ }^{13}$ J. Chen, J. W. Sickler, P. Fendel, E. P. Ippen, F. X. Kärtner, T. Wilken, R. Holzwarth, and T. W. Hänsch, Opt. Lett. 33, 959 (2008).

${ }^{14}$ T. Steinmetz, T. Wilken, C. Araujo-Hauck, R. Holzwarth, T. W. Hänsch, and T. Udem, Appl. Phys. B 96, 251-256 (2009).

${ }^{15}$ A. Haboucha, W. Zhang, T. Li, M. Lours, A. N. Luiten, Y. Le Coq, and G. Santarelli, Opt. Lett. 36, 3654 (2011).

${ }^{16}$ M. Aidelsburger, F. O. Kirchner, F. Krausz, and P. Baum, Proc. Natl. Acad. Sci. U.S.A. 107, 19714-19719 (2010).

${ }^{17}$ A. Gliserin, A. Apolonski, F. Krausz, and P. Baum, New J. Phys. 14, 073055 (2012).

${ }^{18}$ S. Naumov, A. Fernandez, R. Graf, P. Dombi, F. Krausz, and A. Apolonski, New J. Phys. 7, 216 (2005).

${ }^{19}$ B. C. Young, F. C. Cruz, W. M. Itano, and J. C. Bergquist, Phys. Rev. Lett. 82, 3799 (1999).

${ }^{20}$ J. Taylor, S. Datta, A. Hati, C. Nelson, F. Quinlan, A. Joshi, and S. Diddams, IEEE Photon. J. 3, 140 (2011).

${ }^{21}$ W. Zhang, T. Li, M. Lours, S. Seidelin, G. Santarelli, and Y. Le Coq, Appl. Phys. B 106, 301-308 (2012).

${ }^{22}$ T. van Oudheusden, P. L. E. M. Pasmans, S. B. van der Geer, M. J. de Loos, M. J. van der Wiel, and O. J. Luiten, Phys. Rev. Lett. 105, 264801 (2010).

${ }^{23}$ Z. Li, Y. Fu, M. Piels, H. Pan, A. Beling, J. E. Bowers, and J. C. Campbell, Opt. Express 19, B385-B390 (2011).

${ }^{24}$ M. Hentschel, R. Kienberger, Ch. Spielmann, G. A. Reider, N. Milosevic, T. Brabec, P. B. Corkum, U. Heinzmann, M. Drescher, and F. Krausz, Nature 414, 509 (2001).

${ }^{25}$ A. Baltuška, T. Udem, M. Uiberacker, M. Hentschel, E. Goulielmakis, C. Gohle, R. Holzwarth, V. S. Yakovlev, A. Scrinzi, T. W. Hänsch, and F. Krausz, Nature 421, 611 (2003).

${ }^{26}$ R. Kienberger, E. Goulielmakis, M. Uiberacker, A. Baltuška, V. Yakovlev, F. Bammer, A. Scrinzi, Th. Westerwalbesloh, U. Kleineberg, U. Heinzmann, M. Drescher, and F. Krausz, Nature 427, 817 (2004).

${ }^{27}$ P. Baum and A. H. Zewail, Chem. Phys. 366, 2 (2009).

${ }^{28}$ L. Veisz, G. Kurkin, K. Chernov, V. Tarnetsky, A. Apolonski, F. Krausz, and E. Fill, New J. Phys. 9, 451 (2007).

${ }^{29}$ R. P. Chatelain, V. R. Morrison, C. Godbout, and B. J. Siwick, Appl. Phys. Lett. 101, 081901 (2012).

${ }^{30}$ M. Gao, H. Jean-Ruel, R. R. Cooney, J. Stampe, M. de Jong, M. Harb, G. Sciaini, G. Moriena, and R. J. D. Miller, Opt. Express 20, 12048 (2012).

${ }^{31}$ C. Weninger and P. Baum, Ultramicroscopy 113, 145-151 (2012).

${ }^{32}$ D. Kreier and P. Baum, Opt. Lett. 37, 2373 (2012).

${ }^{33}$ F. O. Kirchner, S. Lahme, F. Krausz, and P. Baum, New J. Phys. 15, 063021 (2013). 